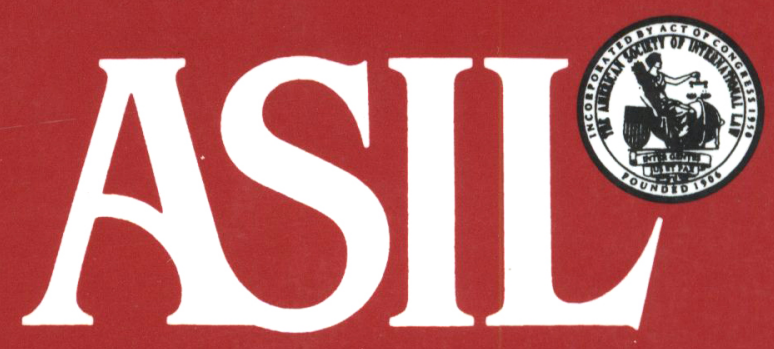

THE AMERICAN SOCIETY OF INTERNATIONAL LAW

Proceedings

of the 90 th

ARE

INSTITUTIONS

DOING THEIR

JOB?

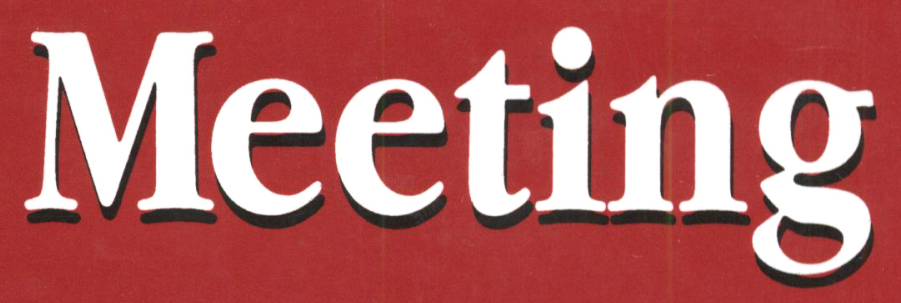

March 27-30, 1996

Washington, D.C. 


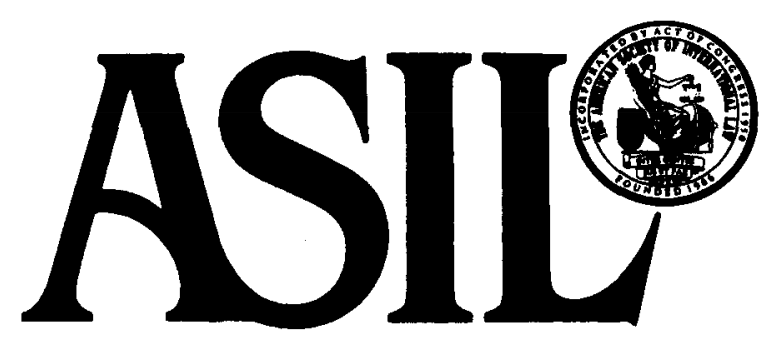

THE AMERICAN SOCIETY OF INTERNATIONAL LAW

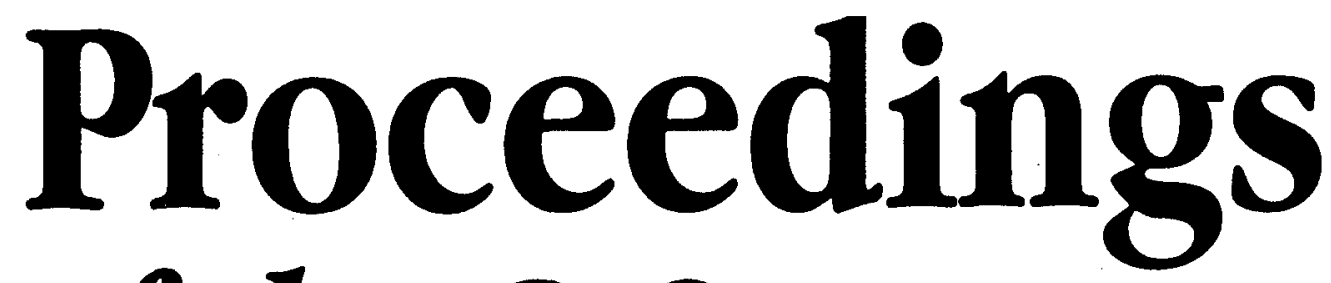

of the 90 th

ARE

INTERNATIONAL

INSTITUTIONS

DOING THEIR

JOB?
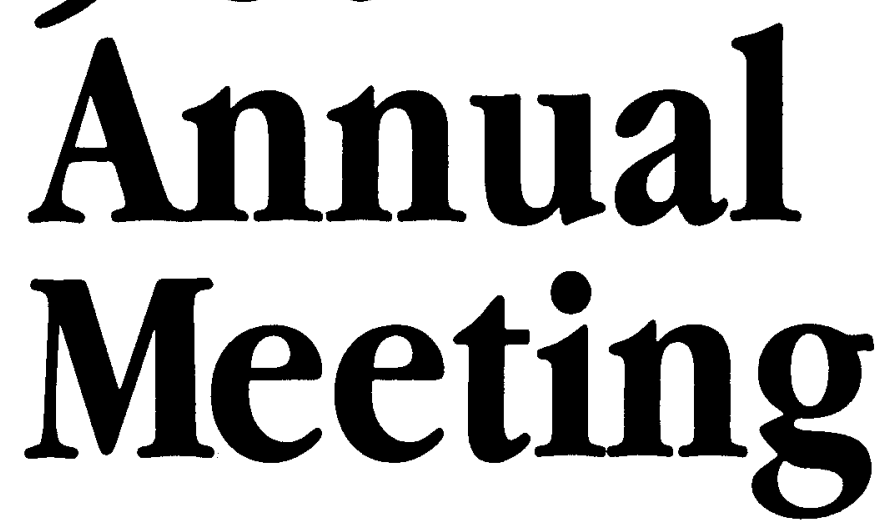

March 27-30, 1996

Washington, D.C. 


\section{The American Society of International Law}

The Society is a professional association devoted to the study and use of law in international affairs. Membership is open to people of all nations, lawyers and nonlawyers, who are interested in the challenging problems of law and policy. Its large and diverse membership of some 4,400, drawn from more than 100 countries, includes scholars, practitioners, government officials, international civil servants, and students.

Founded in 1906, the Society serves as a meeting place, forum and collegial research center, hospitable to all viewpoints in its meetings, publications and other activities. Publications include the American Journal of International Law, International Legal Materials, Studies in Transnational Legal Policy, the ASIL Newsletter, and reports and books produced by its programs of research, study and outreach.

The Society is a nonpartisan, tax-exempt, nonprofit corporation headquartered at Tillar House on Sheridan Circle in Washington, DC.

It is the policy of The American Society of International Law not to take an official position, by resolution or otherwise, upon controversial questions. The views expressed in the addresses, remarks and discussions delivered at its Annual Meetings and appearing in its printed Proceedings are those of the individual speakers and are not to be taken as representing the views of the Society.

\section{Subscription Information}

ASIL members and nonmembers may subscribe to the Proceedings of the Annual Meeting for \$75; outside U.S.A., \$85; or airmail, \$95. To place a subscription or receive information on back issues, please contact The American Society of International Law, 2223 Massachusetts Avenue, N.W., Washington, DC 20008-2864. Telephone (202) 939-6000; FAX (202) 797-7133.

Authorization to photocopy items for internal or personal use (beyond that permitted by Sections 107 and 108 of the U.S. Copyright Law), or for the internal or personal use of specific clients, is granted by The American Society of International Law for users registered with the Copyright Clearance Center (CCC) Transactional Reporting Service, provided that the base fee of $\$ 5.00$ per copy, plus $\$ .25$ per page, is paid directly to CCC, 222 Rosewood Drive, Danvers, MA 01923. For organizations that have been granted a photocopy license by CCC, a separate system of payment has been arranged. The fee code for users of the Transactional Reporting Service is: $0272-5037 / 97, \$ 5.00+.25$. Educational copying is permitted. Please address requests to CCC Academic Permissions Service; (508) 750-8400, fax (508) 750-4744.

\section{Proper Citation}

The Proceedings should be cited according to the following example:

90 ASIL PRoc. 224 (1996).

(C) 1997 The American Society of International Law 


\section{INTRODUCTION}

In announcing the Society's 1996 Annual Meeting, the Meeting's planners invited participants to address a "theme question": "Are International Institutions Doing Their $J o b$ ?" Elaborating, they asked:

In the late twentieth century, what are international institutions? If not limited to permanent, intergovernmental institutions like the United Nations, then what others exist? Do they complement, compete with or supplant each other?

What is demanded or expected or international institutions today?

Are they equipped to respond? Where are financing questions particularly difficult?

What is the political and social context within which international institutions must operate today?

Are there structural weaknessses built into existing institutions which create obstacles to effective performance? What are the expectations raised by sovereignty?

What factors help or hinder effective performance by international institutions-cultural factors and diversity? leadership? law?

Not all of the Meeting's panels were intended to be "theme panels"' charged with responding explicitly to some or all of these inquiries. Fully two-thirds did so, in respect of a widely diverse set of international tasks and concerns and the strikingly varied institutions set up to address them. Of these panels, fully a half-dozen, in keeping with the roiling global milieu of the nineties, dealt with various aspects of collective response to localized crises-security, economic or social (in addition to a somewhat adversarial examination of the Middle East "peace process"). Other panels tackled, with varying degress of comprehensiveness, big pieces of the international community's continuing agenda such as control of nuclear and conventional weaponry; environmental protection; trade, economic integration, and human rights. Others took on more specialized areas of international community business, such as control of transnational crime, the regulation of international capital flows, the evolving role of China in international economic institutions, protection of intellectual property in information technology, coping with official corruption particularly in aspiring democratic societies. And one panel tackles the question of the effectiveness of "non-binding" international rules and principles, institutionally generated with public or private participation.

In the end the best of these discussions turn out to be less concerned with drawing up an explicit job evaluation for the international organizations and other arrangements concerned, than simply with describing - unsentimentally and intelligibly - what they do, how they do it, and the mission they seek to fulfull, and in prescribing new modes of operation better designed to meet newly emerging needs. They offer an abundance of useful information and insight. Collectively they tend to reaffirm and give substance to the notion that international organizations - their policymaking and authorizing procedures, their cadre of experienced bureaucrats, their supporting professional cadres in national political and administrative apparatuses, their capacity for political legitimization-are an indispensable, relatively cost-effective resource for any country with substantial international interests and for the community as a whole.

Of the remaining panels, not obviously intended to fit the "theme," it is perhaps a sign of the pervasiveness of the institutional dimension of contemporary international affairs that scarcely any is devoid of some significant institutional content. But a number are of considerable interest in their own right. To mention only a sample: One panel turns attention to the twentieth centry experience of lawyer-statesmen in the United States. A pair of dispute settlement discussions focuses on current developments in international arbitration and adjudication. For those willing to dig for them, a panel on "Cultural Dominance" will yield some suggestive and stimulating nuggets of insight into problems of multiculturalism and political organization. Many will find a discussion of the implications of elec- 


\section{iv Introduction}

tronic information technology for the discipline and practice of international law informative and exciting. Finally, a similarly informative two-person discussion-cum-debate on the U.S. Helms-Burton sanctions legislation on Cuba draws issues clearly in this sharply controverted matter.

The Proceedings Assistant Editor and I express special thanks to Patricia Allen, who proofread the text with typical competance, and to the Reporters for their indispensable role in producing this volume. Our thanks go also to ASIL interns Kara Zivin and Ryan Whitacre, and to the ASIL Library staff for their assistance.

JohN LAWRENCE HARGROVE Editor

December 1996 


\section{PROCEEDINGS OF THE NINETIETH ANNUAL MEETING OF THE AMERICAN SOCIETY OF INTERNATIONAL LAW}

\section{ARE INTERNATIONAL INSTITUTIONS DOING THEIR JOB?}

Co-Chairs of the Committee on the AnNual MEeting

Arthur Rovine

Ruth Wedgwood
EdITOR OF THE PROCEEDINGS John Lawrence Hargrove AssisTaNT EdITOR Amy Joyce Berks

\section{SUMMARY OF CONTENTS}

International Trade in 1995 and 1996: Review and Preview .......... 1

Global Networks, New Technologies and International Law . . . . . . . . 16

Peace Operations and the War Powers: A Comparative Constitutional

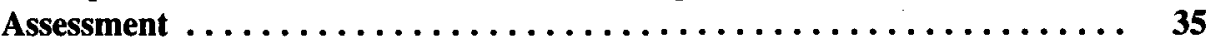

International Litigation: Trends and Developments $\ldots \ldots \ldots \ldots \ldots \ldots \ldots$

Kleptocracy and Democracy $\ldots \ldots \ldots \ldots \ldots \ldots \ldots \ldots \ldots \ldots \ldots$

International Law and Economics . . . . . . . . . . . . . . . . . 109

Isolationism and Internationalism in International Politics $\ldots \ldots \ldots \ldots \ldots$

Lawyers as Statesmen: Twentieth Century U.S. Attitudes Toward International Law .............................. 139

Money Across Borders $\ldots \ldots \ldots \ldots \ldots \ldots \ldots \ldots \ldots \ldots \ldots \ldots \ldots \ldots \ldots 16 \ldots$

Transparency, Accountability and Private Actors $\ldots \ldots \ldots \ldots \ldots \ldots$

Cultural Dominance $\ldots \ldots \ldots \ldots \ldots \ldots \ldots \ldots \ldots \ldots \ldots \ldots \ldots$

Law and Development $\ldots \ldots \ldots \ldots \ldots \ldots \ldots \ldots \ldots \ldots \ldots \ldots \ldots$

International Arbitration $\ldots \ldots \ldots \ldots \ldots \ldots \ldots \ldots \ldots \ldots \ldots \ldots \ldots \ldots$

The Influence of International Human Rights Law on State Courts and State

Constitutions ............................... 259

Emerging Issues in Information Technology $\ldots \ldots \ldots \ldots \ldots \ldots \ldots \ldots \ldots$

International Response to Secessionist Conflicts $\ldots \ldots \ldots \ldots \ldots \ldots \ldots \ldots$

Year of International Law in Review $\ldots \ldots \ldots \ldots \ldots \ldots \ldots \ldots \ldots$

Plenary Address $\ldots \ldots \ldots \ldots \ldots \ldots \ldots \ldots \ldots \ldots \ldots \ldots \ldots \ldots \ldots \ldots$

Plenary Panel: Nonbinding Norms and the Problems of Compliance ...... 348

Cuba and U.S. Sanctions and Extraterritoriality $\ldots \ldots \ldots \ldots \ldots \ldots \ldots$

A Look at Current Action on the Conventional Weapons Convention of

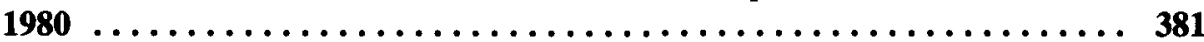

China and International Economic Institutions $\ldots \ldots \ldots \ldots \ldots \ldots \ldots$

World Trade Organization $\ldots \ldots \ldots \ldots \ldots \ldots \ldots \ldots \ldots \ldots \ldots \ldots \ldots \ldots$ 


\section{vi Summary of Contents}

International Financial Institutions' Response to Crises . . . . . . . . . . 428

Women and the United Nations $\ldots \ldots \ldots \ldots \ldots \ldots \ldots \ldots \ldots \ldots \ldots$

Middle East Peace Process $\ldots \ldots \ldots \ldots \ldots \ldots \ldots \ldots \ldots \ldots \ldots \ldots \ldots \ldots \ldots$

Yugoslavia $\ldots \ldots \ldots \ldots \ldots \ldots \ldots \ldots \ldots \ldots \ldots \ldots \ldots \ldots \ldots \ldots \ldots \ldots \ldots$

Environment: New Institutional Challenges $\ldots \ldots \ldots \ldots \ldots \ldots \ldots \ldots \ldots$

International Institutions and Economic Integration $\ldots \ldots \ldots \ldots \ldots \ldots$

ASIL Annual Dinner Address $\ldots \ldots \ldots \ldots \ldots \ldots \ldots \ldots \ldots \ldots \ldots \ldots$

Transnational Crime $\ldots \ldots \ldots \ldots \ldots \ldots \ldots \ldots \ldots \ldots \ldots \ldots \ldots \ldots$

Forced Movement of Peoples $\ldots \ldots \ldots \ldots \ldots \ldots \ldots \ldots \ldots \ldots \ldots \ldots \ldots$

Nuclear Non-Proliferation and Weapons Control $\ldots \ldots \ldots \ldots \ldots \ldots \ldots 5$

Wrap-Up Panel: Are International Institutions Doing Their Job? . . . . . . 583

Violence Against Women and International Law: Rape as a War Crime . . . 605

Violence Against Women and the U.S. Immigration Laws . . . . . . . . 616

Appendices . . . . . . . . . . . . . . . . . . . . . . . . . 627

Index $\ldots \ldots \ldots \ldots \ldots \ldots \ldots \ldots \ldots \ldots \ldots \ldots \ldots \ldots \ldots \ldots \ldots \ldots$ 


\section{PROCEEDINGS OF THE NINETIETH ANNUAL MEETING OF THE AMERICAN SOCIETY OF INTERNATIONAL LAW}

\section{ARE INTERNATIONAL INSTITUTIONS DOING THEIR JOB?}

Co-Chairs of The Committee

on the AnNual Meeting

Arthur Rovine

Ruth Wedgwood
Editor of THE Proceedings John Lawrence Hargrove ASSISTANT EDITOR Amy Joyce Berks

\section{CONTENTS}

International Trade in 1995 and 1996: Review and Preview

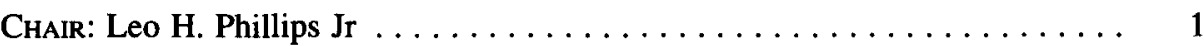

Moderators: Judith H. Bello $\ldots \ldots \ldots \ldots \ldots \ldots \ldots \ldots \ldots \ldots \ldots \ldots \ldots$

Gary N. Horlick ..................... 1

Part I. International Trade in 1995 and 1996: Looking Back and Forward

REMARKS BY:

Daniel K. Tarullo . . . . . . . . . . . . . . . . . . . 1

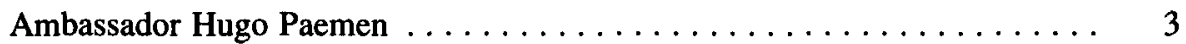

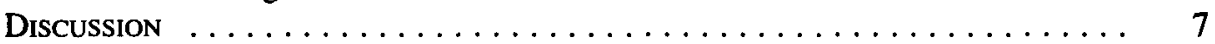

Part II. A 1995 News Hour

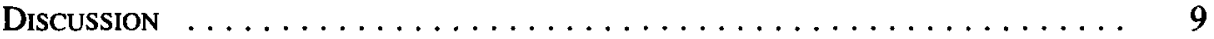

Part III. Segue to 1996: The Polls, Candidates, Advice and Positions on International Trade

REMARKS BY:

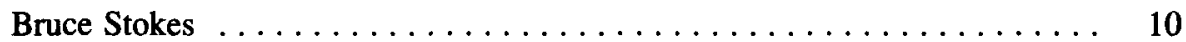

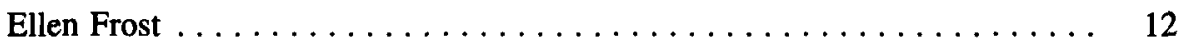

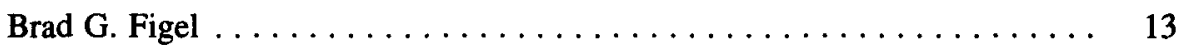

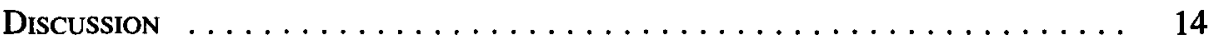

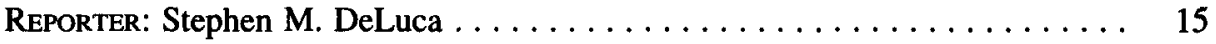

Global Networks, New Technologies and International Law

ChaIR: John King Gamble $\ldots \ldots \ldots \ldots \ldots \ldots \ldots \ldots \ldots \ldots \ldots \ldots \ldots \ldots$

INTRODUCTORY REMARKS BY:

John King Gamble . . . . . . . . . . . . . . . . . . . . . . 16

Successful Networking in a Related Field: The CIESIN Example.

Roberta Balstad Miller ......................... 16

Bringing the World to the Classroom: Computers as Masters or Slaves?

Donald E. Buckingham ....................... 20

Baby, Bathwater, Computers, Networks and Shakespeare: The ASIL in 2007.

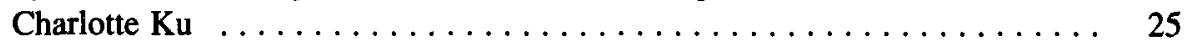

Environmental Treaties and Resource Indicators. Frederick Zimmerman . . . . 28

The ASIL Home Page on the World Wide Web. Jill McC. Watson ... . . . . 31

Taking Stock-What Does it All Mean? Detlev F. Vagts . . . . . . . . . 32

REPORTER: Manuel Terranova ........................ 34

Peace Operations and the War Powers: A Comparative Constitutional Assessment

Chatr: R. James Woolsey $\ldots \ldots \ldots \ldots \ldots \ldots \ldots \ldots \ldots \ldots \ldots \ldots$

INTRODUCTORY REMARKS BY:

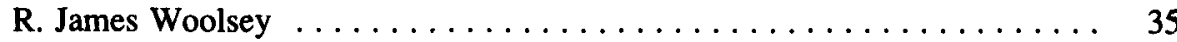


Is There a General Trend in Constitutional Democracies Toward

Parliamentary Control Over War-and-Peace Decisions?

Lori Fisler Damrosch . . . . . . . . . . . . . . . . . . . . 36

REMARKS BY:

Richard Debobes ........................... 40

Hisashi Owada ............................. 42

The Russian Experience. Bakhtiyar Tuzmukhamedov ............. 44

The War Powers in French Constitutional Law. Elisabeth Zoller . . . . . . . . 46

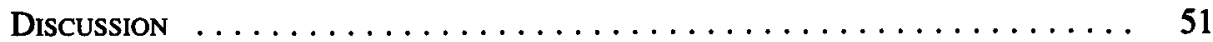

REPORTER: Christopher A. Ford $\ldots \ldots \ldots \ldots \ldots \ldots \ldots \ldots \ldots \ldots \ldots \ldots, 61$

\section{International Litigation: Trends and Developments}

Chatr: Lawrence W. Newman . . . . . . . . . . . . . . . 62

Forum Non-Conveniens, Comity, Antisuit Injunctions and Parallel

Proceedings. Nancy Nelson . . . . . . . . . . . . . . . . . 62

Obtaining Evidence in the United States for Foreign Proceedings.

Lawrence W. Newman ............................ 67

Developments in International Human Rights Litigation. Barton Legum . . . . . 71

Conciliation, Mediation and Dispute Resolution. Ronald E.M. Goodman . . . . 75

Discussion .............................. 78

REPORTER: Jonathan Clark Green $\ldots \ldots \ldots \ldots \ldots \ldots \ldots \ldots \ldots \ldots$

\section{Kleptocracy and Democracy}

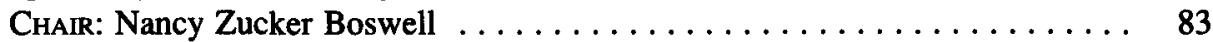

Corruption and Democracy. Susan Rose-Ackerman . . . . . . . . . . . . 83

Organized Crime and Corruption in Russia: Implications for the United States

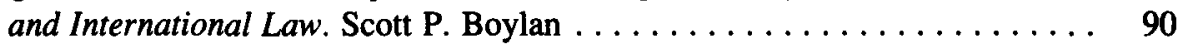

REMARKS BY:

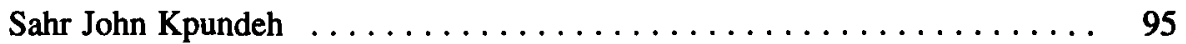

Katherine Voltz .......................... 97

Combating Corruption: Are International Institutions Doing Their Job?

Nancy Zucker Boswell ......................... 98

Discussion .................................. 105

REPORTER: Jane Weaver . . . . . . . . . . . . . . . . . . . . . . . . . . . . . . 107

\section{International Law and Economics}

Chair: Alan Sykes . . . . . . . . . . . . . . . . . . . . . . . . 109

REMARKS BY:

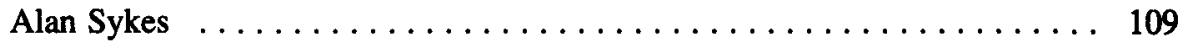

Warren Schwartz ........................... 111

Frank H. Buckley . . . . . . . . . . . . . . . . . . 113

Paul Stephan $\ldots \ldots \ldots \ldots \ldots \ldots \ldots \ldots \ldots \ldots \ldots \ldots \ldots \ldots \ldots \ldots$

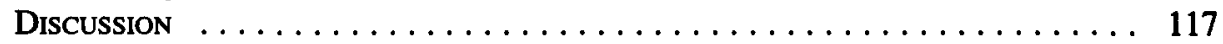

RePORTER: Charlene Denise Oliveira Cabral . . . . . . . . . . . . . 122

Isolationism and Internationalism in International Politics

CHAIR: Mark Clodfelter . . . . . . . . . . . . . . . . . . . . . . . . . . 123

REMARKS BY:

Mark Clodfelter . . . . . . . . . . . . . . . . . . . . . . 123 
Howard L. Berman . . . . . . . . . . . . . . . . . . . . 125

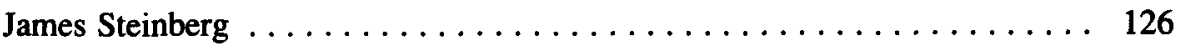

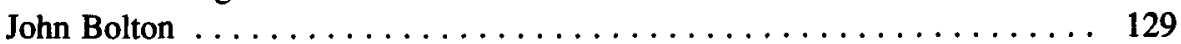

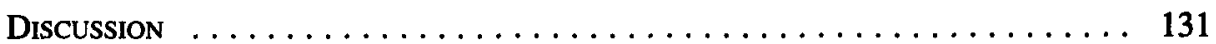

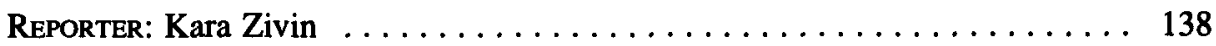

\section{Lawyers as Statesmen: Twentieth Century U.S. Attitudes}

Toward International Law

ChaIR: Frederic L. Kirgis . . . . . . . . . . . . . . . . . . . . . . . . . . 139

Elihu Root, James Brown Scott and the Early Years of the ASIL.

Frederic L. Kirgis . . . . . . . . . . . . . . . . . . . . . 139

Charles Evans Hughes as International Lawyer. Richard D. Friedman . . . . . 143

American Judicial Internationalism in the Twentieth Century.

Michael Dunne . . . . . . . . . . . . . . . . . . . . . . 148

Dean Rusk and International Law. Thomas J. Schoenbaum . . . . . . . . . . 155

Discussion .................................. 158

REPORTER: Kathleen Bergin $\ldots \ldots \ldots \ldots \ldots \ldots \ldots \ldots \ldots \ldots$

\section{Money Across Borders}

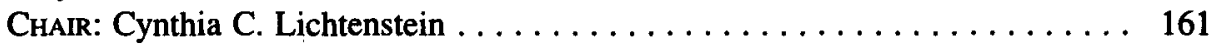

REMARKS BY:

Cynthia C. Lichtenstein $\ldots \ldots \ldots \ldots \ldots \ldots \ldots \ldots \ldots \ldots \ldots \ldots$

International Capital Flows in the 1990s: The Role of Institutional Investors.

Jane W. D'Arista . . . . . . . . . . . . . . . . . . . . . . 164

REMARKS BY:

Avinash Persaud . . . . . . . . . . . . . . . . . . . . . . . . . . . 169

Capital Movements Under the Maastricht Treaty. Patrick Juillard . . . . . . 172

Discussion .............................. 175

Transparency, Accountability and Private Actors

Chalr: Lee A. Kimball . . . . . . . . . . . . . . . . . . . . . . 178

The International Organization for Standardization and the Drafting of Private

Environmental Standards. Naomi Roht-Arriaza .............. 178

REMARKS BY:

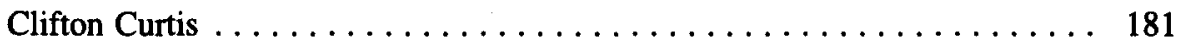

Transparency and Form. Patrick O. Gudridge .............. 183

REMARKS BY:

Lee A. Kimball $\ldots \ldots \ldots \ldots \ldots \ldots \ldots \ldots \ldots \ldots \ldots \ldots \ldots \ldots \ldots$

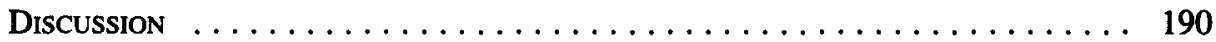

REPORTER: Pinelopi Makrodimitris $\ldots \ldots \ldots \ldots \ldots \ldots \ldots \ldots \ldots \ldots \ldots$

\section{Cultural Dominance}

Chatr: Winston P. Nagan . . . . . . . . . . . . . . . . . . . . . 193

Toward a General Legal Theory About Cultural Dominance: Law, Power and

Group Deprivations. Winston P. Nagan . . . . . . . . . . . . . . . . . . . 193

A Note on Multiculturalism and Cultural Dominance. Yael Tamir . . . . . . . 200

Powersharing as a Response to Cultural Dominance. David Wippman ..... 206

The Self-Determination of Minorities and Sphere Sovereignty.

Johan D. van der Vyver . . . . . . . . . . . . . . . . . 211

Nationalism "Good" and "Bad"': The Vicissitudes of an Obsession.

Nathaniel Berman 


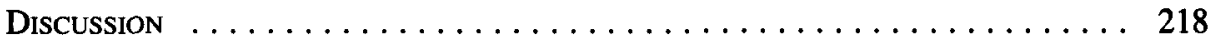

RePORTER: David R. Penna . . . . . . . . . . . . . . . . . . . . . . 221

\section{Law and Development}

ChaIr: David M. Trubek . . . . . . . . . . . . . . . . . 223

Law and Development: Then and Now. David M. Trubek . . . . . . . . . 223

Company Law for Emerging Markets: The Case of Russia. Bernard Black . . . 226

Law and Development: Toward a Pluralist Vision. Mohan Gopalan Gopal . . . 231

Law and Development in Russia: A Misguided Enterprise? Kathryn Hendley . . 237

Discussion . . . . . . . . . . . . . . . . . . . . . . . . . . . . . . 240

REPORTER: Rumu Sarkar . . . . . . . . . . . . . . . . . . . . . . . . . . . . 243

\section{International Arbitration}

ChaIr: Charles Brower . . . . . . . . . . . . . . . . . . . . . 244

INTRODUCTORY REMARKS BY:

Charles Brower . . . . . . . . . . . . . . . . . . . . . . . . . . 244

RoundTABle PARTICIPANTS:

Lucy Reed . . . . . . . . . . . . . . . . . . . . . . . . . 245

Howard Holtzmann $\ldots \ldots \ldots \ldots \ldots \ldots \ldots \ldots \ldots \ldots \ldots \ldots \ldots \ldots$

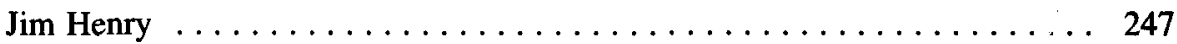

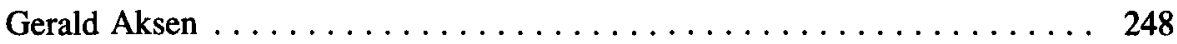

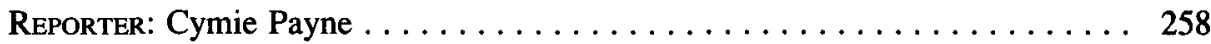

The Influence of International Human Rights Law on State Courts and State Constitutions

ChaIR: Alice H. Henkin . . . . . . . . . . . . . . . . . . . . 259

INTRODUCTORY REMARKS BY:

Alice H. Henkin . . . . . . . . . . . . . . . . . . . . . . . 259

REMARKS BY:

Christine Durham ................................. 259

The Preemptive and Interpretive Force of International Human Rights Law in State Courts. Joan Fitzpatrick . . . . . . . . . . . . . . . . . . . . 262

REMARKS BY:

Paul Hoffman . . . . . . . . . . . . . . . . . . . . . . . 266

Herman Schwartz . . . . . . . . . . . . . . . . . . . . . . . 269

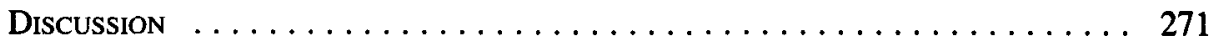

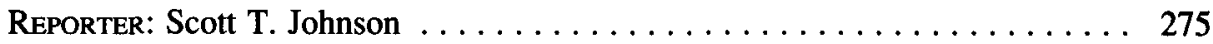

\section{Emerging Issues in Information Technology}

CHAIR: Anthony D'Amato . . . . . . . . . . . . . . . . . . . . 276

REMARKS BY:

Jerome Reichman . . . . . . . . . . . . . . . . . . . 276

Virginia Wise ............................... 284

The Problem of Developing an International Protection Standard in Today's Multicultural, Economically Diverse, Global Marketplace.

Doris Estelle Long . . . . . . . . . . . . . . . . . . . . . . . . . 286

Discussion . . . . . . . . . . . . . . . . . . . . . . . . . . . 292

REPORTER: Debra Jean Hamilton . . . . . . . . . . . . . . . . . . . . . 295

International Response to Secessionist Conflicts

ChaIR: Nicholas Kittrie 
INTRODUCTORY REMARKS BY:

Nicholas $\mathbf{N}$. Kittrie . . . . . . . . . . . . . . . . . . . . . . . . 296

REMARKS BY: Hurst Hannum . . . . . . . . . . . . . . . . . 297

Is There a Right to Secede? Yoram Dinstein . . . . . . . . . . . . . . . 299

Negotiating the Settlement of Secessionist Conflicts.

Tom Farer . . . . . . . . . . . . . . . . . . . . . . . 303

REMARKS BY:

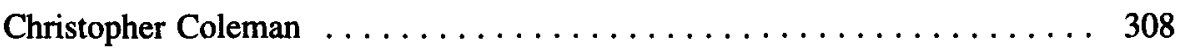

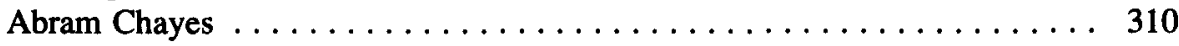

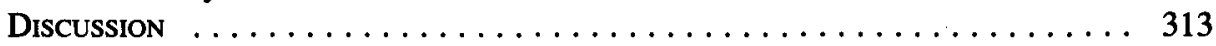

REPORTER: Kate Greene $\ldots \ldots \ldots \ldots \ldots \ldots \ldots \ldots \ldots \ldots \ldots \ldots \ldots$

\section{Year of International Law in Review}

CHAIR: Enid C.B. Schoettle . . . . . . . . . . . . . . . . . . . . . 319

The United States Financial Veto. Jose E. Alvarez . . . . . . . . . . . . . . 319

The United Nations in Crisis: Reflections from within the Secretariat.

Hans Corell . . .......................... 324

Some Changes in International Administrative Justice. Hubert Thierry . . . . . 329

International Tribunal for Rwanda. Brenda Sue Thornton . . . . . . . . 333

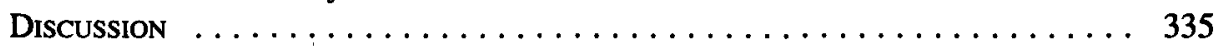

REPORTER: Suneeta Dewan $\ldots \ldots \ldots \ldots \ldots \ldots \ldots \ldots \ldots \ldots \ldots$

\section{Plenary Address}

Fifty Years of the World Court: A Critical Appraisal. Stephen M. Schwebel . . 339

\section{Plenary Panel: Nonbinding Norms and the Problems of Compliance}

INTRODUCTORY REMARKS BY:

Edith Brown Weiss . . . . . . . . . . . . . . . . . . . 348

The Changing Nature of International Obligations: Effectiveness of

International Law and Compliance. Naoya Okuwaki . . . . . . . . . . . 348

International Actors and Compliance: How Important is Casting to a Good

Box Office? Dinah Shelton . . . . . . . . . . . . . . . 353

Development Cooperation and Compliance With International Environmental

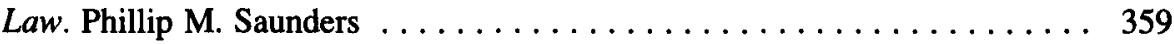

CommentaRy By: Antonia Handler Chayes ............... 365

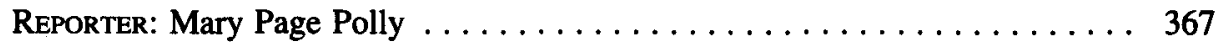

Cuba and U.S. Sanctions and Extraterritoriality

INTRODUCTORY REMARKS BY:

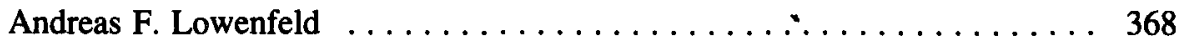

Title III of the Helms-Burton Act Does Not Violate International Law.

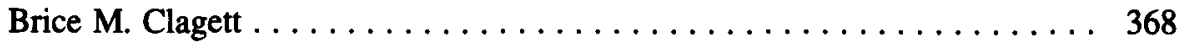

REMARKS BY:

Andreas F. Lowenfeld . . . . . . . . . . . . . . . . . . . . . . . . 373

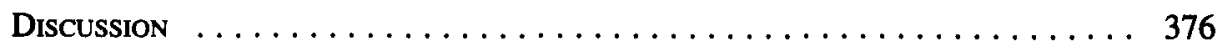

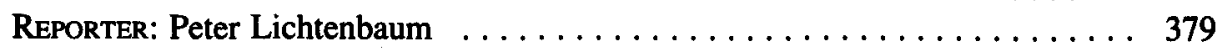

A Look at Current Action on the Conventional Weapons Convention of 1980

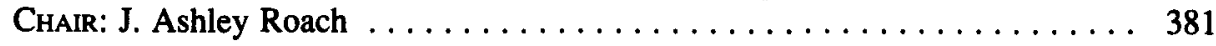

INTRODUCTORY REMARKS BY:

J. Ashley Roach . . . . . . . . . . . . . . . . . . . . 


\section{xii Contents}

Current Action of the Conventional Weapons Convention of 1980.

Michael J. Matheson . . . . . . . . . . . . . . . . . . . . . . . . . 381

Land Mines: Humanitarian Concerns, Law and Politics. Robert Rydberg . . . 384

REMARKS BY:

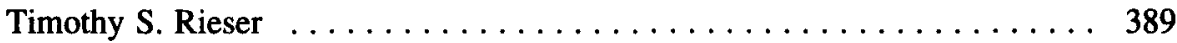

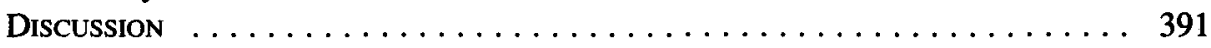

REPORTER: Shirley E. Whitfield $\ldots \ldots \ldots \ldots \ldots \ldots \ldots \ldots \ldots \ldots \ldots$

\section{China and International Economic Institutions}

Chair: James V. Feinerman . . . . . . . . . . . . . . . . . . . . . . . . . 397

China and the World Bank. Natalie Lichtenstein . . . . . . . . . . . . . . . . . 397

China's Quest to Enter the GATT/WTO. James V. Feinerman . . . . . . . 401

REMARKS BY:

James V. Feinerman . . . . . . . . . . . . . . . . . . 405

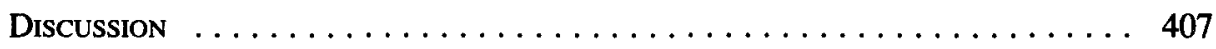

REPORTER: Bing Ling $\ldots \ldots \ldots \ldots \ldots \ldots \ldots \ldots \ldots \ldots \ldots \ldots \ldots$

\section{World Trade Organization}

ChaIR: Amelia Porges $\ldots \ldots \ldots \ldots \ldots \ldots \ldots \ldots \ldots \ldots \ldots . \ldots \ldots$

INTRODUCTORY REMARKS BY:

Amelia Porges . . . . . . . . . . . . . . . . . . . . 412

Legal Developments in the WTO. William J. Davey ... . . . . . . . 415

The WTO: Is it Working? Jeffrey M. Lang . . . . . . . . . . . . . . 419

REMARKS BY:

John H. Jackson . . . . . . . . . . . . . . . . . . . . . . . . . . . 423

Discussion .................................. 424

REPORTER: Matthew P. Jaffe . . . . . . . . . . . . . . . . . . . . . . . . 427

\section{International Financial Institutions' Response to Crises}

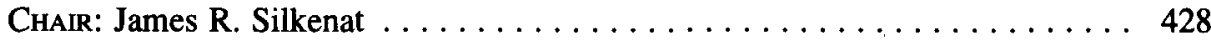

INTRODUCTORY REMARKS BY:

James R. Silkenat . . . . . . . . . . . . . . . . . . . . . . 428

Rebuilding War-Torn Countries: Role of the International Financial Institutions. Nicole Ball . . . . . . . . . . . . . . . . . . . 428

The Bretton Woods Institutions: Coping With Crises. Herbert Morais . . . . . . 433

REMARKS BY:

Alvaro de Soto . . . . . . . . . . . . . . . . . . . . 439

Discussion $\ldots \ldots \ldots \ldots \ldots \ldots \ldots \ldots \ldots \ldots \ldots \ldots \ldots \ldots \ldots \ldots \ldots . \ldots \ldots 42$

REPORTER: Louis J. Ross $\ldots \ldots \ldots \ldots \ldots \ldots \ldots \ldots \ldots \ldots \ldots \ldots 44$

\section{Women and the United Nations}

Chalr: Elizabeth F. Defeis . . . . . . . . . . . . . . . . . 445

REMARKS BY:

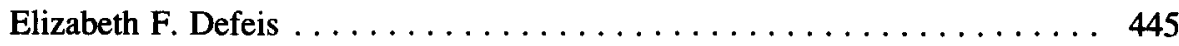

Surita Sandosham . . . . . . . . . . . . . . . . . . . . . . 448

The UN Role in the Promotion of Gender Equality. Rosario Green . . . . . . 454

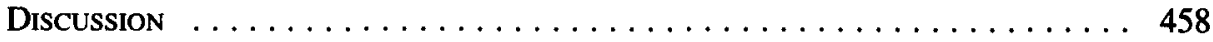

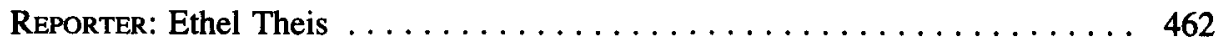

Middle East Peace Process

CHAIR: J.C. Hurewitz . . . . . . . . . . . . . . . . . . . . . . 463 
REMARKS BY:

Hasan Rahman . . . . . . . . . . . . . . . . . . . . . 463

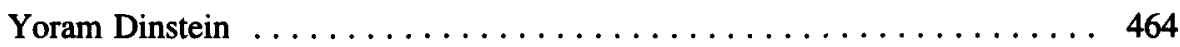

Hasan Rahman ............................ 466

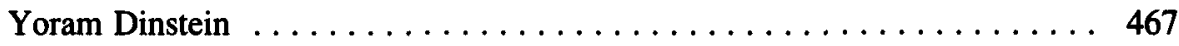

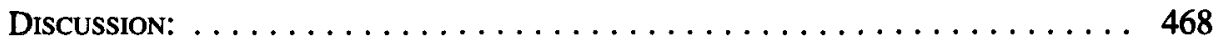

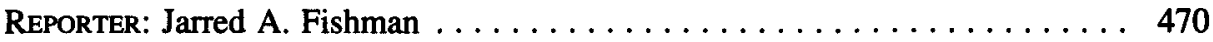

\section{Yugoslavia}

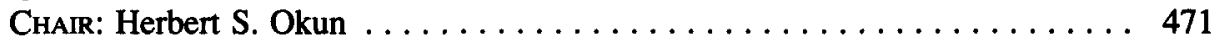

INTRODUCTORY REMARKS BY:

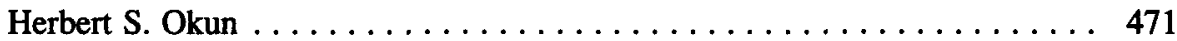

REMARKS BY:

Susan Woodward .........................471

Larry Johnson . . . . . . . . . . . . . . . . . . . . 474

The Bosnian Constitution: The Road to Dayton and Beyond. Paul C. Szasz . . 479 REMARKS BY:

Theodor Meron ...........................4 483

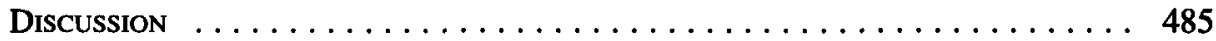

RePORTERs: Amy J. Berks and Kara Zivin . . . . . . . . . . . . . . . . . 487

\section{Environment: New Institutional Challenges}

ChaIR: Said Mahmoudi $\ldots \ldots \ldots \ldots \ldots \ldots \ldots \ldots \ldots \ldots \ldots$

INTRODUCTORY REMARKS BY:

Said Mahmoudi .............................4 488

A New Fish Story: Improve Fishery Conservation and Management Through International Agreement. David Balton . . . . . . . . . . . . . 490

Global Emissions Trading: Lessons from Domestic Law and Arms Control.

Richard Williamson ............................4493

International Institutions and the World's Water. Phillip R. Trimble . . . . . 499

Discussion ................................ 504

REPORTER: Steven T. Mines $\ldots \ldots \ldots \ldots \ldots \ldots \ldots \ldots \ldots$. . . . . . . . . 507

International Institutions and Economic Integration

ChaIR: Frederick M. Abbott . . . . . . . . . . . . . . . . . . . . 508

REMARKS BY:

Frederick M. Abbott $\ldots \ldots \ldots \ldots \ldots \ldots \ldots \ldots \ldots \ldots \ldots \ldots \ldots$

Charlene Barshefsky . . . . . . . . . . . . . . . . . . . . . 511

Mercosur: A Pragmatic Regional Building Block. Marcos

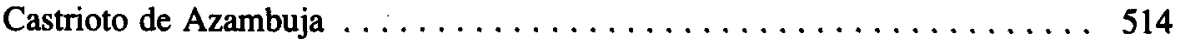

International Institutions and European Integration. Jochen Abr. Frowein . . . 516

Discussion ............................ 518

REPORTER: Andreas R. Ziegler $\ldots \ldots \ldots \ldots \ldots \ldots \ldots \ldots \ldots \ldots \ldots \ldots . \ldots \ldots$

ASIL Annual Dinner Address

Who Needs Ambassadors? Challenges to American Diplomacy Today.

Richard N. Gardner . . . . . . . . . . . . . . . . . . . . . 525

\section{Transnational Crime}

Chair: Bruno A. Ristau 
International Cooperation and Transnational Organized Crime.

Ugljesa Zvekic

REMARKS BY:

Mary Ellen Warlow . . . . . . . . . . . . . . . . . . . . . . 537

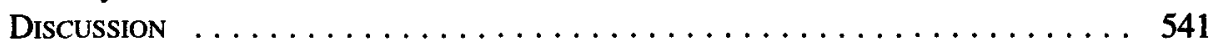

REPORTER: Debra Jean Hamilton . . . . . . . . . . . . . . . . 544

\section{Forced Movement of Peoples}

ChAIR: Jean-Marie Henckaerts

INTRODUCTORY REMARKS BY:

Jean-Marie Henckaerts . . . . . . . . . . . . . . . . . . 545

The Legal Dimensions of Preventing Forced Migration. Arthur C. Helton . . . 546

The Question of a Right Not to be Displaced. Maria Stavropoulou . . . . . . . . 549

REMARKS BY:

Luke T. Lee . . . . . . . . . . . . . . . . . . . . . . . . . . . 554

Discussion $\ldots \ldots \ldots \ldots \ldots \ldots \ldots \ldots \ldots \ldots \ldots \ldots \ldots \ldots \ldots \ldots \ldots \ldots$

REPORTER: George E. Little $\ldots \ldots \ldots \ldots \ldots \ldots \ldots \ldots \ldots \ldots \ldots \ldots$

\section{Nuclear Non-Proliferation and Weapons Control}

ChaIR: Mary Elizabeth Hoinkes $\ldots \ldots \ldots \ldots \ldots \ldots \ldots \ldots \ldots \ldots \ldots$

INTRODUCTORY REMARKS BY:

Mary Elizabeth Hoinkes $\ldots \ldots \ldots \ldots \ldots \ldots \ldots \ldots \ldots \ldots . \ldots \ldots$

REMARKS BY:

Ambassador Jayantha Dhanapala . . . . . . . . . . . . . . . . . . . 567

David A. Koplow . . . . . . . . . . . . . . . . . . . . . 570

Martha Caldwell Harris . . . . . . . . . . . . . . . . . . . . . . . . . . 574

John B. Rhinelander . . . . . . . . . . . . . . . . . . . . . . . 576

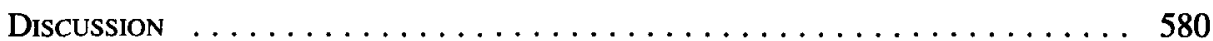

REPORTER: Lila J. Bakke $\ldots \ldots \ldots \ldots \ldots \ldots \ldots \ldots \ldots \ldots \ldots \ldots \ldots$

\section{Wrap-Up Panel: Are International Institutions Doing Their Job?}

ChalR: Harold K. Jacobson $\ldots \ldots \ldots \ldots \ldots \ldots \ldots \ldots \ldots \ldots \ldots \ldots$

INTRODUCTORY REMARKS BY:

Harold K. Jacobson . . . . . . . . . . . . . . . . . . . . . 583

The ASIL as an Epistemic Community. Charlotte Ku . . . . . . . . . . . 584

What, Exactly, Is the Job of International Institutions? Brigitte Stern . . . . . . 585

Are International Organizations Doing Their Job? International Legislation.

Elihu Lauterpacht . . . . . . . . . . . . . . . . . . . . . . . . . . . . . 593

The Success and Failure of International Organizations.

By Thomas M. Franck . . . . . . . . . . . . . . . . . 596

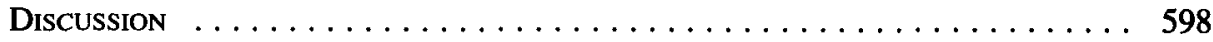

REPORTER: Susan B. Hunt . . . . . . . . . . . . . . . . . . . . 604

Violence Against Women and International Law: Rape as a War Crime

ChaIR: Miriam Sapiro $\ldots \ldots \ldots \ldots \ldots \ldots \ldots \ldots \ldots \ldots \ldots$

INTRODUCTORY REMARKS BY:

Miriam Sapiro . . . . . . . . . . . . . . . . . . . . . . . . . . . . . 605

Arriving at Rwanda: Extension of Sexual Assault Prosecution Under the

Statutes of the Ad Hoc International Criminal Tribunals.

Patricia Viseur Sellers 
Discussion $\ldots \ldots \ldots \ldots \ldots \ldots \ldots \ldots \ldots \ldots \ldots \ldots \ldots \ldots \ldots \ldots \ldots$

REPORTER: Julia Hall $\ldots \ldots \ldots \ldots \ldots \ldots \ldots \ldots \ldots \ldots \ldots \ldots$

Violence Against Women and the U.S. Immigration Laws

CHAIR: Nancy Goodman . . . . . . . . . . . . . . . . 616

REMARKS BY:

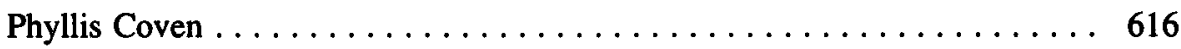

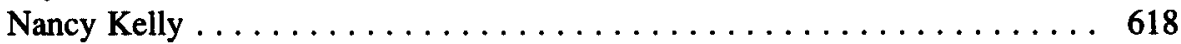

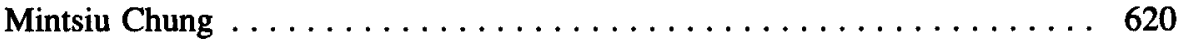

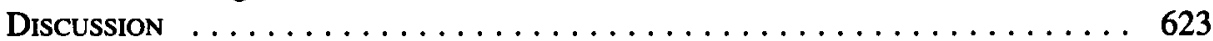

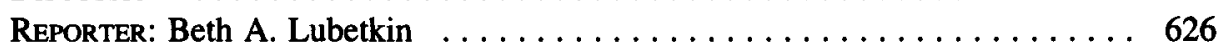

Appendices $\ldots \ldots \ldots \ldots \ldots \ldots \ldots \ldots \ldots \ldots \ldots \ldots \ldots \ldots \ldots \ldots$

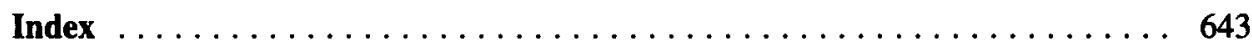

